\title{
A New Equivalent Circuit Extraction Method For Quasi-static Regions
}

\author{
Benjamin D. Braaten*, Robert M. Nelson, and Yuxin Feng \\ North Dakota State University, Department of Electrical and \\ Computer Engineering, Fargo, ND 58105, email: benbraaten@ieee.org.
}

Abstract - A new method for calculating the equivalent circuit of a quasi-static region based on scattered fields is presented. Two problems with a quasi-static region are chosen to validate the procedure. It is then shown that the geometry in the quasi-static region can be modeled accurately in Mininec as an equivalent circuit and that the computation times with the equivalent circuit are significantly less.

\section{Introduction}

Recently, numerically stable electric field integral equations (EFIE) were developed [1] to evaluate electromagnetic scattering problems that may contain both electrically small geometrically complex and electrically large regions. Although the EFIE derived in [1] have shown improvements in computational efficiency it has been noticed by Olsen [2] that problems with a large number of quasi-static regions can take a significant amount of time to evaluate. This has led to some development of modeling these quasi-static regions with equivalent circuits [1], [2]. These equivalent circuits can then be defined in efficient fast full-wave solvers that do not evaluate complex dielectric regions such as Mininec [3]. This will then result a much shorter computation time for very complex problems.

In this work we introduce a method, based on work in [4], for determining the equivalent circuit of a quasi-static region. This method can then be used to determine the equivalent circuit of many different types of geometries, including geometries that may not provide a convenient analytical method for evaluating the equivalent circuit. Two problems are chosen to validate the method presented here. The first is a dipole with a capacitively dominant quasi-static region similar to the one shown on the left side in Figure $1 \mathrm{a}$ ). The second is a scattering problem with two dielectric insulators located along the length of a long wire. The geometry in this case does not allow for a convenient analytical equivalent circuit. Finally, a few comments will be made about the computation times of problems without equivalent circuits and problems with equivalent circuits.

\section{Computing the equivalent circuit}

To introduce the procedure for calculating the equivalent circuit of the quasi-static region we start with a thin-wire dipole problem. It has been shown [4] that the input impedance of a dipole can be determined by providing an incident field and measuring the resulting scattered fields for three different loads placed at the port. Then the following expression can be used to calculate the input admittance $Y_{D}$ of the dipole [4]:

$$
Y_{D}=-\frac{E_{s}-E_{1}}{E_{o}-E_{1}} G_{o}
$$


where $E_{s}$ is the field due to a short circuit at the port, $E_{o}$ is the field due to an open circuit at the port and $E_{1}$ is the field due to a known port load $R_{o}=1 / G_{o}$. We can see that all the values on the right side of (1) are known values for the problems presented in [4], thus leaving the only unknown $Y_{D}$ to be evaluated. Notice that we can also use (1) to determine $R_{o}$ if we know the scattered fields and the input admittance $Y_{D}$ of the dipole. Solving for $R_{o}$ is the idea behind the equivalent circuit method presented in this communication. To start we solve for $R_{o}$ in (1) in the following manner

$$
Z_{o}=-\frac{E_{s}-E_{1}}{E_{o}-E_{1}} Z_{D}
$$

where $Z_{o}$ has replaced $R_{o}$ to generalize the problem and $Z_{D}$ is the input impedance of the antenna with $Z_{D}=1 / Y_{D}$. We will now use (2) to calculate the equivalent circuit for the quasi-static region of interest. For illustration we chose the geometry on the left side of Figure 1 a) as our quasi-static region connected to an arbitrary antenna. We have also defined a thin-wire test dipole on the right in Figure 1 a) with a known length $L$ and radius $a$. The port of the test dipole is defined by the dashed circle. This then gives us an antenna with a well-known input impedance [3] and can be used in (2) as $Z_{D}$. Next, the quasi-static region of interest is redefined at the port of the test dipole and the entire problem is excited by an incident plane wave. This then induces currents on every surface and results in a scattered field $E_{q, s}$. This field will then be used in (2) as $E_{q, s}=E_{1}$. Next, the quasi-static region at the port of the test dipole is modified to act as an open and a short and the resulting scattered fields for both cases are used in (2) as $E_{o}$ and $E_{s}$, respectfully. This then defines all the unknown fields and (2) can be used to calculate the equivalent circuit of the quasi-static region. It should be noted that the field values in (2) are far-field values. Next, we use the new EFIE in [1] to evaluate the three scattering problems associated with $E_{1}, E_{o}$ and $E_{s}$. To do this a moment method (MOM) based graphical user's interface (GUI) was developed in Matlab to evaluate axially symmetric problems [1]. This GUI is called QUICNEC which stands for QUasi-static Inductive Capacitive Numerical Electromagnetics Code. The new EFIE evaluated by QUICNEC are not summarized here but a very thorough discussion can be found in [1].

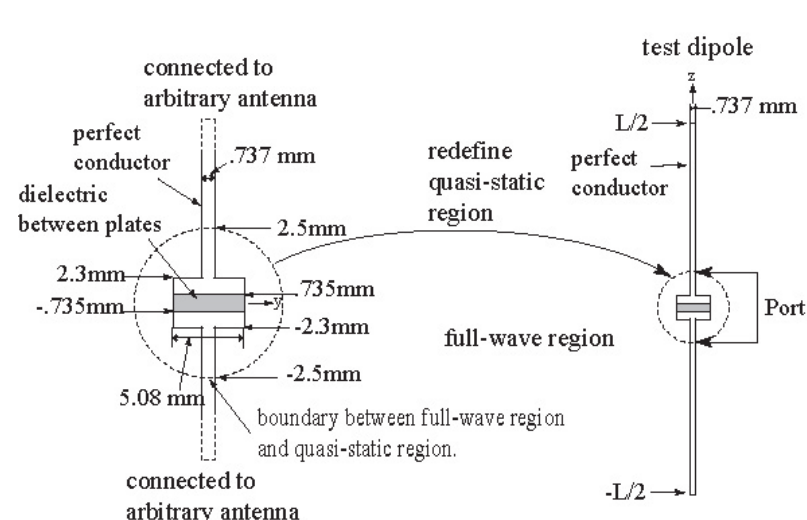

a)

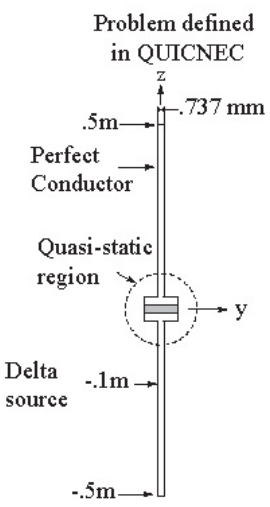

b)

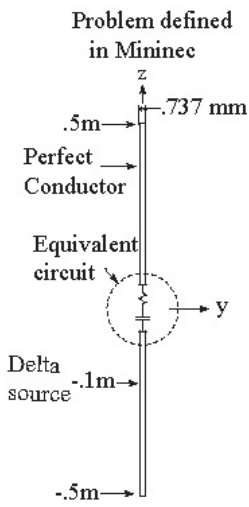

c)

Figure 1: (a) Equivalent circuit extraction method; (b) validation problem defined in QUICNEC; (c) validation problem defined in Mininec. 


\section{Examples}

In this section we start by using (2) to calculate the equivalent circuit of the quasistatic region on the left in Figure 1 a). The quasi-static region is similar to the geometry found in work by Olsen [2] which investigates problems that have capacitively dominant quasi-static regions. The quasi-static region has two conducting plates separated by a dielectric and is symmetric about the origin. By redefining the quasi-static region at the port of the test dipole on the right in Figure 1 a) and defining $\mathrm{L}=.3 \mathrm{~m}$ we evaluate the problem in QUICNEC and use (2) to calculate an equivalent circuit at $1 \mathrm{GHz}$. This resulted in an equivalent resistance of $R_{s, e q} \approx 0 \Omega$ and an equivalent capacitance of $C_{s, e q}=.32 p F$ for a relative permittivity $\varepsilon_{r}=2.23$ and relative permeability $\mu_{r}=1$. The equivalent circuit is shown in Figure 2 a) (insert). Because of the nice geometry in the capacitive quasi-static region a comparison with an analytical method can be made. This can be done by using $C_{a, e q}=\varepsilon_{0} \varepsilon_{r} A / d$ where $A$ is the area of the plate and $d$ is the distance between the plates. Then for the geometry on the left in Figure $1 \mathrm{a}$ ) it is determined that $C_{a, e q}=.28 p F$. This result confirms that (2) can be used to extract accurate equivalent circuits.

An entirely new problem is defined in QUICNEC and Mininec to compare the accuracy of the equivalent circuit calculated above. First the entire dipole in Figure $1 \mathrm{~b}$ ) is defined in QUICNEC with the quasi-static region on the left in Figure 1 a) defined at the origin. The quasi-static region is symmetric about the y-axis and has the same dimensions that are defined in Figure 1 a). Instead of exciting the problem with an incident field a delta source is defined at $-.1 \mathrm{~m}$. This source is then used to evaluate the input impedance $Z_{i n}$ of the dipole at -.1m. Note that this is not the input impedance of the test dipole $Z_{D}$ in (2) (or Figure 1 a)). Similarly, the dipole in Figure $1 \mathrm{c}$ ) is defined in Mininec and also excited by a delta source for two cases: when $C_{s, e q}$ and $C_{a, e q}$ are used for the lumped capacitor that represents the quasi-static region. The imaginary part of the input impedance $Z_{i n}$ at $-.1 \mathrm{~m}$ is shown in Figure 2 a) for all three cases. We can see that the values computed by the EFIE in QUICNEC compare very well with the two equivalent circuits defined and calculated in Mininec. The real part was also shown to have good agreement. Also, for each frequency point calculated in Figure 2 a) QUICNEC had a computation time of 57 seconds and Mininec had a shorter computation time of $<1$ second.

The last problem investigated was an insulator made out of a dielectric with the geometry in Figure 2 b) (insert) [5]. The dielectric was defined with $\mu_{r}=1$ and $\varepsilon_{r}=2$. Using a test dipole in QUICNEC an equivalent resistance of $R_{s, e q}=1251 \Omega$ and equivalent capacitance of $C_{s, e q}=.00692 p F$ was calculated at $500 \mathrm{MHz}$. Then the insulator was placed at two positions (at $-1 \mathrm{~m}$ and $1 \mathrm{~m}$ ) along a 6 meter wire with a radius of $1 \mathrm{~mm}$. The actual physical structure was used in QUICNEC and the equivalent circuits were used in Mininec. An incident field of $1340 \mathrm{KHz}$ was defined to excite both problems. A comparison of the induced full-wave currents is shown in Figure $2 \mathrm{~b}$ ) and good agreement is shown to exist. Also, for the calculations in Figure $2 \mathrm{~b}$ ) QUICNEC had a computation time of 6 minutes and Mininec had a much shorter computation time of $<1$ second. It should also be mentioned that a problem with a quasi-static region that represents an inductor similar to the one in [1] has also been evaluated successfully and will be shown at the time of presentation. 


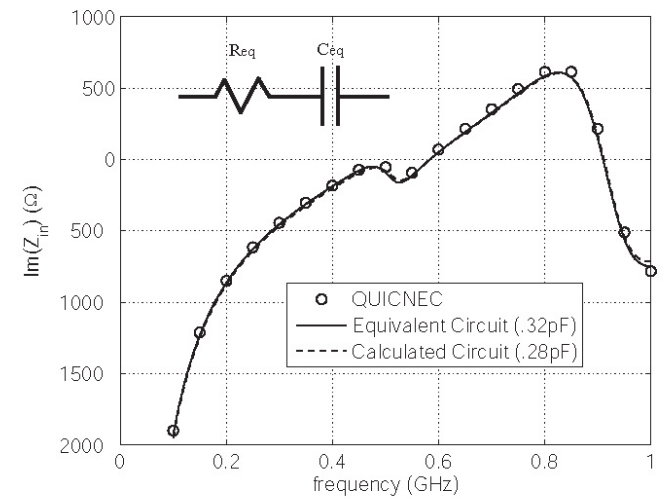

a)

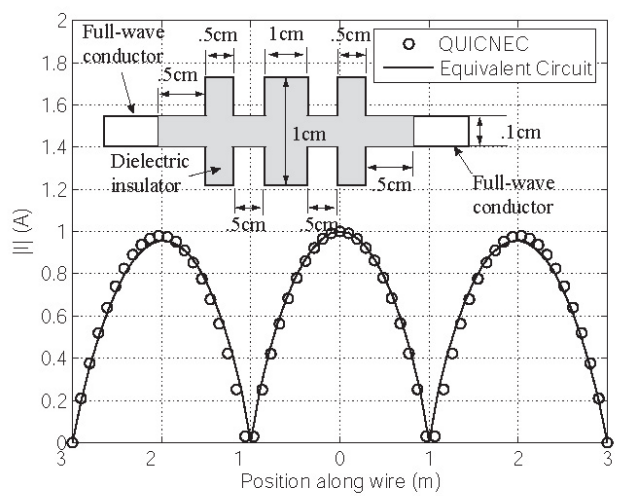

b)

Figure 2: (a) Imaginary part of input impedances for capacitively loaded dipole; (b) magnitude of induced current along $6 \mathrm{~m}$ wire.

\section{Conclusion}

A method for computing the equivalent circuit of a quasi-static region based on scattered fields and newly derived EFIE has been presented. The equivalent circuits for two representative problems have been evaluated. Very good agreement is shown between computations obtained when EFIE are used to evaluate the entire problem and those obtained when the equivalent circuits are used in Mininec. Computation times between the EFIE and Mininec are compared and it is shown that a significant reduction in computation time can be realized in Mininec once the equivalent circuit is determined.

\section{References}

[1] B.D. Braaten, R.M. Nelson and M.A. Mohammed, "Electric field integral equations for electromagnetic scattering problems with electrically small and electrically large regions," IEEE Transactions on Antennas and Propagation, Vol. 56, No. 1, January 2008, pp. 142-150.

[2] Robert G. Olsen, G.L. Hower, and P.D. Mannikko, "A Hybrid Method for Combining Quasi-Static and Full-Wave Techniques for Electromagnetic Scattering Problems," IEEE Transactions on Antennas and Propagation, Vol. 36, No. 8, pp. 1180-1184, August 1988.

[3] J.W. Rockway and J.C. Logan, "The New MININEC (Version 3): A MiniNumerical Electromagnetics Code," U.S. Department of Commerce National Technical Information Service, Springfield, VA, September 1986, pp. 1-21.

[4] J.T. Mayhan, A.R. Dion and A.J. Simmons, "A technique for measuring antenna drive port impedance using backscatter data," IEEE Transactions on Antennas and Propagation, Vol. 42, No. 4, April 1994, pp. 526-533.

[5] www.universal-radio.com/catalog 